\title{
Human Fall Detection using Maximum Euclidean Distance and Ellipse Approximation
}

\author{
Dujtep Thawonsusin and Pranchalee Samanpiboon
}

\begin{abstract}
The population of senior citizens is continuously increasing, and most of them are living alone at home. The fall is main cause to make severe injury or mortality. The fall detection systems turn into equipment to help elderly rapidly. A technology to detect fall based on vision systems offers a new promising solution to analyze human behavior and detect fall events. In this paper, we propose a new method to detect falls in a video sequence. In our proposed system, the Maximum Euclidean Distance was used for human motion analysis. Then the fall posture of human is detected by using the approximated ellipse method. Our algorithm provides promising results on video sequences of daily activities taken by IP camera installed on a high position like bird's eye view and simulated falls with occlusions.
\end{abstract}

Keywords-Fall detection, Shape Context Matching, Euclidean distance.

\section{INTRODUCTION}

With the growing population of elderly and most of them living alone, in-home facility system has intelligent surveillance system due to its promising ability in helping the elderly. Many studies have indicated that falls in elderly people are one of the most dangerous situations at home. When an elderly person is living alone and has a fall accident, he/she is lying on the floor for a long time without any help. Fall detection system can send notification to helper for immediate rescue.

In the recent years, fall detection research is mostly focused on vision-based technology. 3D human tracking was developed using multi-camera [1] to detect diversity of fall actions. However, this method is expensive and difficult to use in real life. Later on, a position human tracking using fish eye camera [2] analyze orientation ellipse of human and the camera installed on top view position. However, the top view position of the camera can make an error when human lies down on a sofa. A 3D depth-sensing camera [3], it can give a good result to detect fall, but the installed camera on side view will be obstructed by the object. Next, human shape estimate by fit ellipse and Motion History Image (MHI) [4] classified by Hidden Markov Models (HMM) give a good result. However, MHI feature is difficult to detection falls with occlusion. 3D modeling to classify human posture [5] gives a good result, but 3D modeling of the human is too complicated to use.

Dujtep Thawonsusin and Pranchalee Samanpiboon are with Department of Control system and Instrumentation Engineering, King Mongkut's University of Technology Thonburi, Bangkok, Thailand.
In this research, we used a single camera which is installed on a high position like bird's eye view to grab the image on a large area. We proposed fall detection system based on Maximum Euclidean Distance and approximated ellipse method through surveillance video sequences. This method can detect human shape from incomplete background subtraction with occlusion of human shape.

\section{SYSTEM OVERVIEW}

A behavior of fall can be found by a large movement and some changes in human posture. For human posture during normal activity, the human shape will gradually change. While during a fall, the human shape will change rapidly. Our fall detection system detected a large movement of human by using Maximum Euclidean Distance. And approximated ellipse analyzed the posture of human to classify fall and nonfall posture in image sequences. The flowchart of our system is shown in Fig. 1.

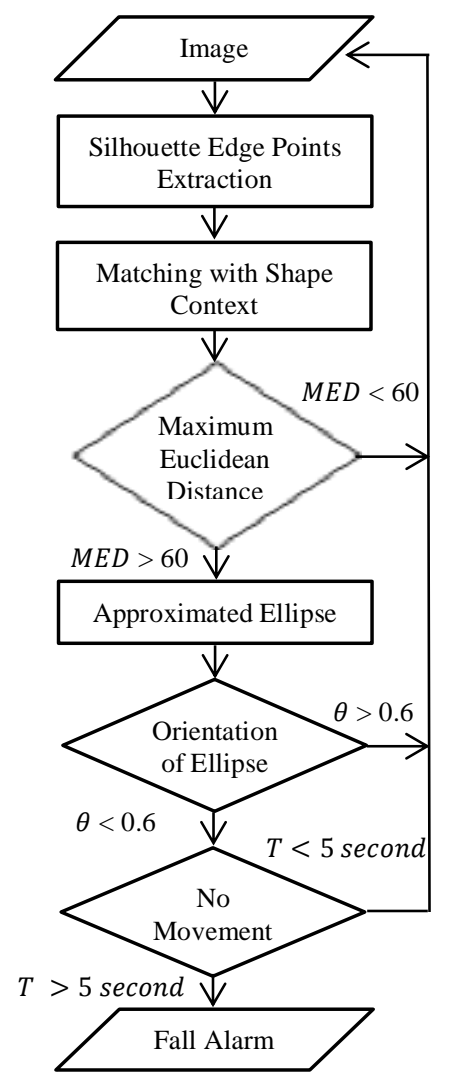

Fig.1: Flowchart of fall detection system 


\section{A. Silhouette Edge Points Extraction}

Usually, the silhouette of the person is used for shape analysis. To extract this silhouette, we use median filtering method [6] to subtract background of the scene and the moving person is then detected by finding the difference image between the incoming frames with the background model. Although median filter background subtraction method is a non-recursive technique and cannot remove shadows, this method requires less processing power. An example of background subtraction is shown in Fig. 2(b).

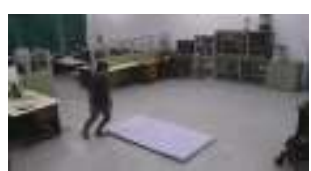

(a)

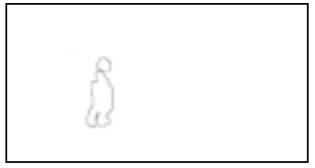

(c)

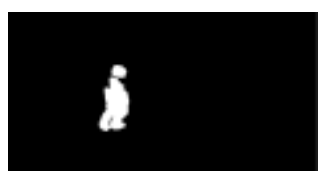

(b)

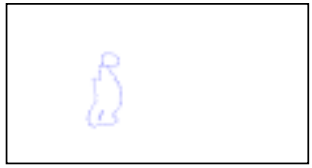

(d)
Fig. 2: (a) Original Image, (b) background subtraction, (c) Canny edge detector, (d) selected edge point.

In the low-cost of IP cameras, there is noise in images. The silhouette segmented from the background subtraction is not clean enough to be used for shape analysis, so we need to remove noise with Morphological operation. After remove noise, we chose to extract some edge point with Canny edge detector. An example of Canny edge detector is shown in Fig. 2(c). We selected only some edge points of the shape because the edge points of a shape are sensitive to human movement. We do not need all points to match consecutive of human shape. The $N$ landmarks are sampled from the edge points on each silhouette for human-shape approximation ( $N$ was 150 for our experiment). The sampling interval, inc, of selected edge points is calculated by equation (1).

$$
\text { inc }=\frac{\max (n b E d g e P t s 1, n b E d g e P t s 2)}{N}
$$

Where $n b E d g e P t s 1$ and $n b E d g e P t s 2$ are the numbers of edge points of the previous frame and the current frame from silhouette image respectively. The selected edge points of image are shown in Fig. 2(d).

\section{B. Matching with Shape Context}

From two images sequences, we used Shape Context [7], [9] to find the best matching points between current and previous human shape. In this research, we used the eighth previous image from the current image for matching process.

In shape matching process, a point $\left(q_{j}\right)$ of the current shape was matched to each point $\left(p_{i}\right)$ of the previous shape for the best corresponding pairs. A log-polar histogram $h_{i}$ was computed to find relative coordinates points. The computation of log-polar histogram is shown in equation (2).

$$
h_{i}=\#\left\{q \neq p_{i}:\left(q-p_{i}\right) \in \operatorname{bin}(k)\right\}
$$

The log-polar histogram gives a position of the polar coordinate system on each selected edge point $p_{i}$ as shown in Fig. 3.

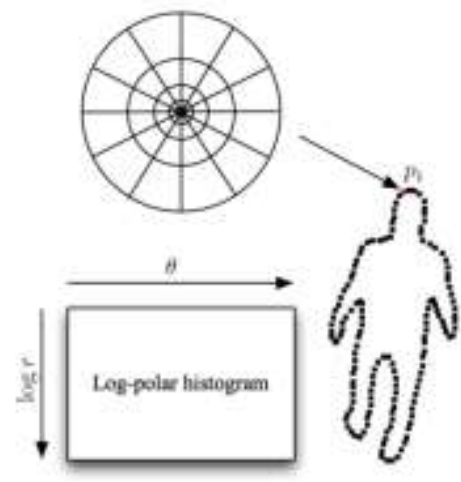

Fig.3: Computation Log-polar histogram of point $p_{i}$. The Log-polar histogram has 5 bins for $\log r$ and 12 bins for $\theta$ [9]

To find similarity points of human shapes in two images sequences, a matching cost $C\left(p_{i}, q_{j}\right)$ for each a pair of points $\left(p_{i}, q_{j}\right)$ was computed by the chi-square test $\left(\chi^{2}\right)$ statistic shown in equation (3).

$$
C\left(p_{i}, q_{i}\right)=\frac{1}{2} \sum_{k=1}^{K} \frac{\left[h_{i}(k)-h_{j}(k)\right]^{2}}{h_{i}(k)+h_{j}(k)}
$$

Where $h_{i}(k)$ and $h_{j}(k)$ represent the K-bin histograms with $p_{i}$ and $q_{i}$ respectively.

From the set of costs $C\left(p_{i}, q_{j}\right)$ between all pairs of points, the best matched-points can find by using the Hungarian algorithm for minimizing the total matching cost $H(\pi)$ given a transition $\pi(i)$ shown in equation (4).

$$
H(\pi)=\sum_{i} C\left(p_{i}, q_{\pi(i)}\right)
$$

The Hungarian algorithm [10] can provide the solution for bipartite matching. The square cost matrix $C\left(p_{i}, q_{i}\right)$ is the input of this algorithm and correspond point to the transformation $\pi(i)$ minimizing $H(\pi)$.

The selected edge points have some bad landmark because of bad segmentation and partial occlusion. There are some outliers in the matching process. The outliers will be removed before last computation of matching. We show example of two images sequences in Fig. 4(a) and 4(b). The selected edge points of two images sequences are show in Fig. 4(c) and 4(d) of previous image and current image respectively. The result of Shape Context Matching from two images sequences are show in Fig. 4(e).

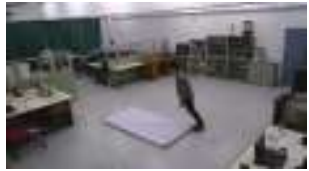

(a)

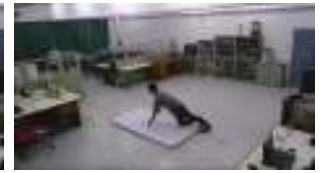

(b) 


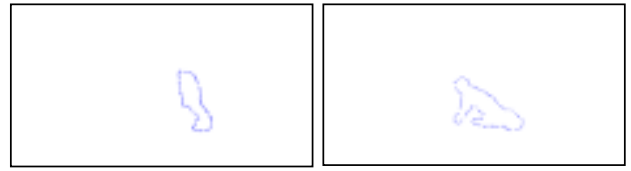

(c)

(d)

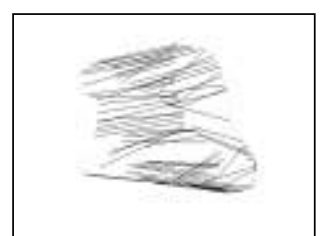

(e)

Fig. 4: Shape Context matching example: (a) (b) Two image sequences, (c) (d) the correspond selected edge points and (e) the matching result

\section{Maximum Euclidean Distance}

From two selected edge points of current image $q_{i}$ and previous image $p_{i}$, we find a large movement to detect fall with Maximum Euclidean Distance [8].

This method classifies abnormal activity from normal activities in all the video sequence. The Maximum Euclidean Distance is shown in equation (5).

$$
\operatorname{MED}(p, q)=\max \sqrt{\sum_{i=1}^{n}\left(q_{i}-p_{i}\right)^{2}}
$$

$q_{i}$ are points on the current shape and $p_{i}$ are points on the previous shape. We find a maximum distance by equation (5) for a large movement calculation to detect fall from the consecutive shapes. Result of Maximum Euclidean Distance calculation is shown in Fig. 5.

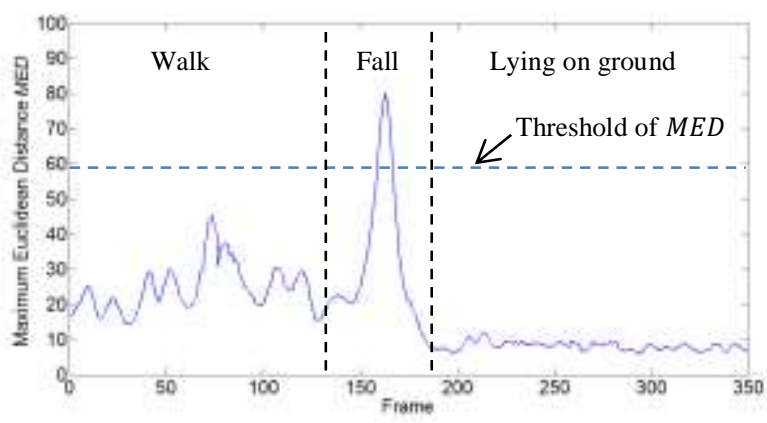

Fig. 5: Example of Maximum Euclidean Distance

\section{Ellipse Approximation Method}

We use Ellipse Approximation method to analyze the changing of human shape in current shape.

An approximated ellipse is defined by the center of ellipse $(\bar{x}, \bar{y})$, its orientation $\theta$ and the length $a$ and $b$ of its major and minor semi-axes.

From the continuous image $f(x, y)$, movement in the current image is given by equation (6).

$$
m_{i j}=\int_{-\infty}^{+\infty} \int_{-\infty}^{+\infty} x^{i} y^{j} f(x, y) d x d y \quad ; i, j=0,1,2 .
$$

The center of the ellipse is obtained by computing the coordinate of the center of mass with first-order and the zeroorder moments: $\bar{x}=m_{10} / m_{00}, \bar{y}=m_{01} / m_{00}$

The centroid $(\bar{x}, \bar{y})$ is used to compute central moment in equation (7).

$$
\mu_{i j}=\int_{-\infty}^{+\infty} \int_{-\infty}^{+\infty}(x-\bar{x})^{i}(y-\bar{y})^{j} f(x, y) d x d y
$$

The angle between major axis of the human shape and horizontal $x$ axis of image gives the orientation of ellipse, and can be computed from the second order of central moment as shown in equation (8).

$$
\theta=\frac{1}{2} \arctan \left(\frac{2 \mu_{11}}{\mu_{20} \mu_{02}}\right)
$$

The approximated ellipse provides information about the shape and orientation of the person in the current image. Fig. 6 shows example of the approximated ellipse of the person 6(a) and the calculated result during fall down 6(b). The orientation angle of changed ellipse when human falls down. In this experiment, the mattress was used to protect the person during fall simulation.

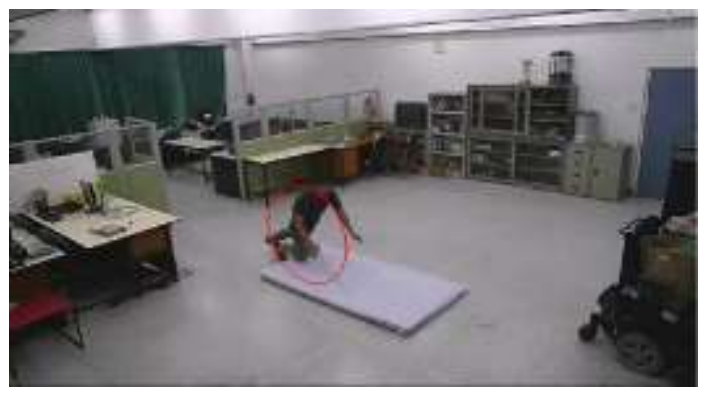

(a)

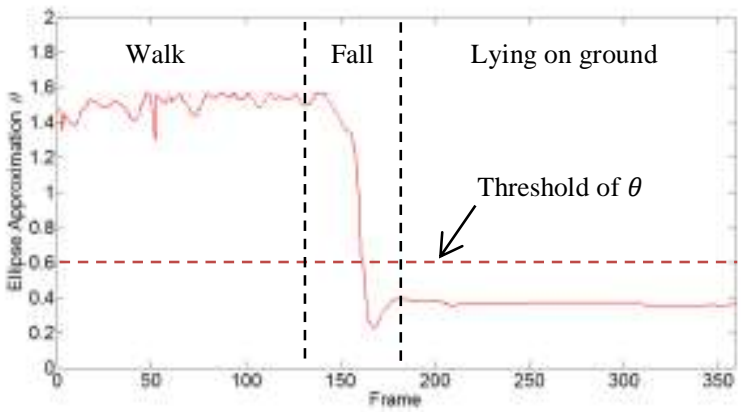

(b)

Fig. 6: (a) Approximated Ellipse of human shape, (b) Result of human fall down

\section{FALL RECOGNITION}

Our fall detection system is based on Maximum Euclidean Distance $(M E D)$ and orientation of ellipse $(\theta)$. Maximum Euclidean Distance quantifies movement of the person to detect large motion like falls, but some behavior of human can also be a large motion such as run and fast bend. Hence, we have to analyze further to discriminate a fall from a normal movement with the orientation of ellipse. This method can detect a change of human in current shape when the human fall. We have three conditions to confirm falls as follow: 
1) If value of Maximum Euclidean Distance is higher than threshold $(M E D>60)$, this indicates that large motion is detected. Condition 2 will be checked in the next step.

2) If value of the orientation of ellipse is lower than the threshold $(\theta<0.6 \mathrm{rad})$, then fall may occur.

3) This last step is used to justify that the falls occur. If no motion under the conditions, $M E D<20$ and $\theta<0.6$, is detected for 5 seconds, the fall detection system will send notification to helper.

The fall motion is confirmed with three mentioned conditions. The red line and blue line represent $\theta$ and $M E D$ respectively. The red dashed line represents threshold value of $\theta$ and blue dashed line represents threshold value of $M E D$.

When all of these three conditions are met, then we can ensure that the fall occurs. The example of considering three conditions to indicate the occurrence of fall is shown in Fig. 7.

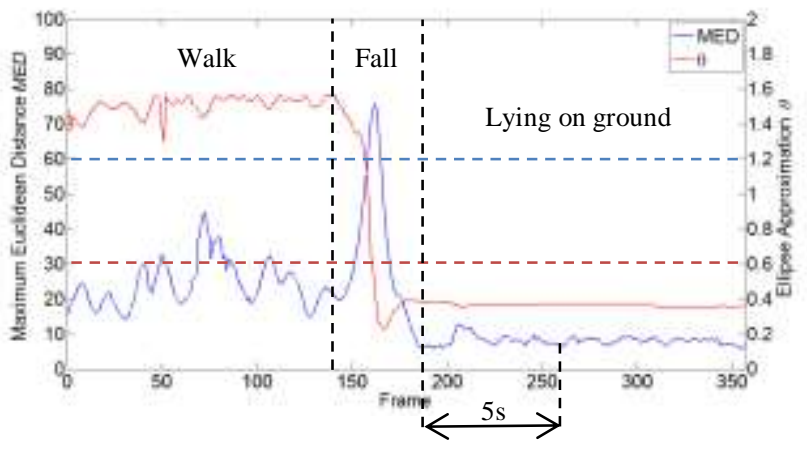

Fig. 7: Example of considering three conditions

\section{EXPERIMENT}

Our fall detection system is a low-cost system with IP camera (WV-SP105) installed on a high position like bird's eye view for getting a wide angle view, about 70 degree angles to grab large area of the room. The image size is $640 \times 480$ pixels and the frame rate is $15 \mathrm{fps}$. In this system, we implemented in Matlab®.

Our data set includes 80 video sequences representing daily normal activities (walking, sitting, bending and lying) and abnormal activities (forward falls, backward falls and falls with occlusion). All these sequences captured the person who entering the room, and some sequences show fall with the occlusion in Fig. 8.

Fig. 8(a) is shown sequence image of fall with occlusion and Fig. 8(b) is result of fall with occlusion. Fall with occlusion is difficult to detect fall because some parts of the human shape are concealed with the object. The Maximum Euclidean Distance calculation can find a large movement of human shape although some parts of the human shape have disappeared. From the experiment, this method is robust with $50 \%$ occlusion of human shape.

Fig. 9(a) is shown example of approximation ellipse. Fig. 9(b) is shown result example of run. The value of Maximum Euclidean Distance is higher than the threshold value (Condition 1 is met). However, the value of orientation of

ellipse is not lower than the threshold value, thus no fall occurs.

The experiment result of fall detection system is shown in Table. I. The result indicates a high performance of fall detecting with $97.61 \%$ sensitivity and non-fall detection with $89.47 \%$ specificity.

\section{CONCLUSION}

In this research, we proposed a new method of fall detection which can recognize fall from normal activities by using Maximum Euclidean Distance (MED) and ellipse approximation method. And the three conditions is used to confirm a fall. The first condition indicates a large motion of human, the second detects a posture of human. The last condition checks the movement after fall occurs, if no movement is detected, the fall will be confirmed and the notification will be sent to helper. Furthermore, our fall detection system was proven its robustness on realistic situation for human fall detection with occlusion from other normal activities.
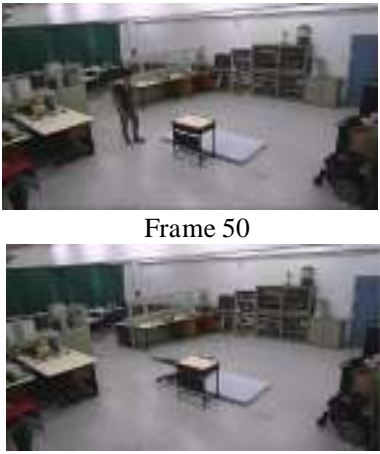

Frame 90

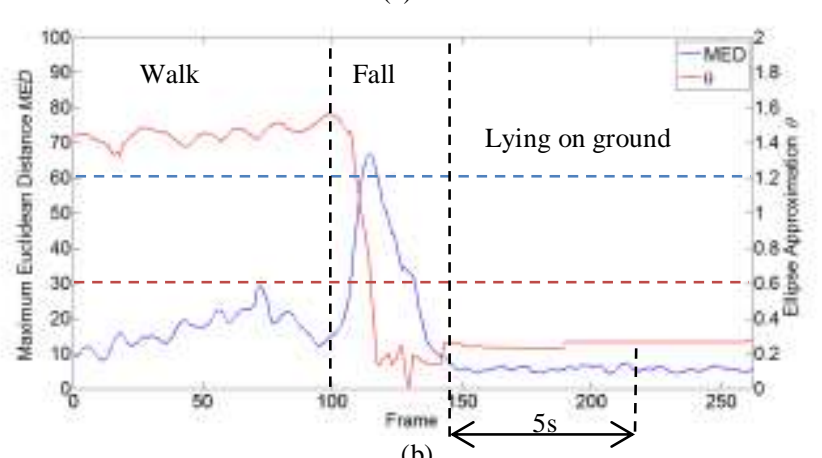

(b)

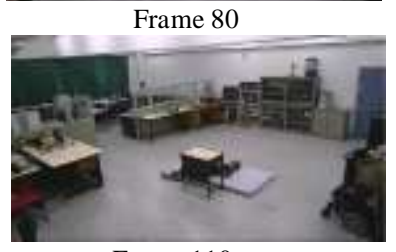

Frame 110

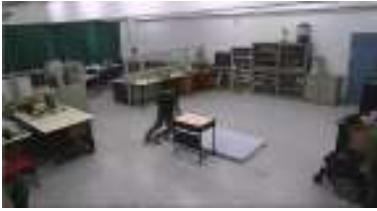

(a)

Fig. 8: (a) Example of fall with occlusion, (b) Result of fall with occlusion

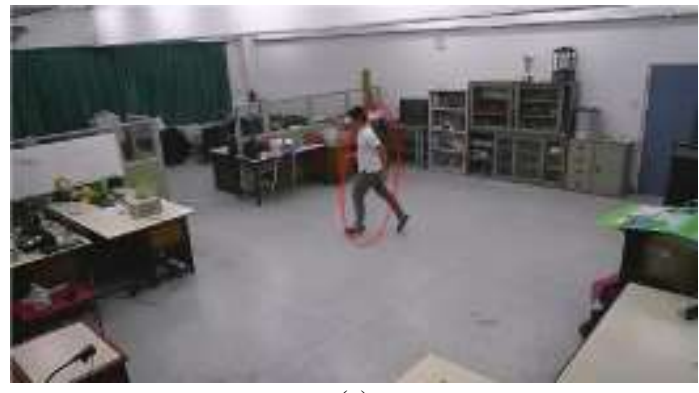

(a) 


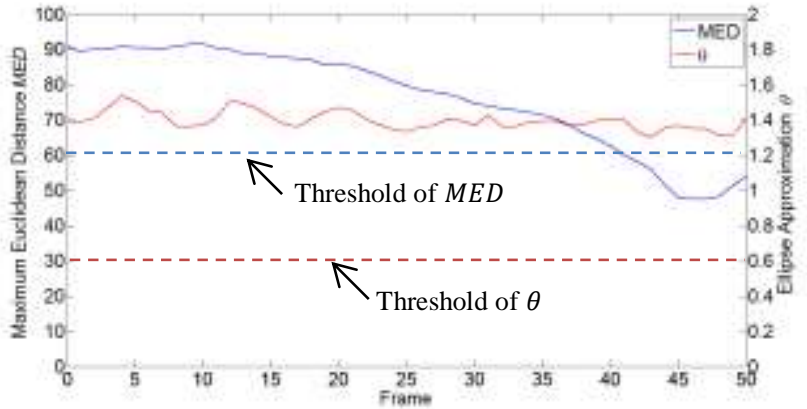

(b)

Fig. 9: (a) Example of run, (b) Result of run

TABLE I: EXPERIMENT RESULT

\begin{tabular}{|c|c|c|}
\hline $\begin{array}{l}\text { System Fall incident } \\
\text { recognition }\end{array}$ & Fall & Non-fall \\
\hline Detection as fall & 41 & 4 \\
\hline Detected as non-fall & 1 & 34 \\
\hline
\end{tabular}

\section{REFERENCES}

[1] S. Zambanini, J. Macbajdik, and M. Kampel, "Detecting falls at Homes Using a Network of Low-Resolution Cameras," Information Technology and Applications in Biomedicine, pp. 1-4, Nov 2010.

[2] R. Hartmann, F. Machot, P. Mahr, and C. Bobda, "Camera-Based System for Tracking and Position Estimation of Humans," Design and Architectures for Signal and Image Processing, pp. 62-67, Oct 2010.

[3] Z. Zhang, W. Liu, V. Metsis, and V. Athitsos, "A ViewpointIndependent Statistical Method for Fall Detection," International Conference on Pattern Recognition, pp. 3626 - 3630, Nov 2012.

[4] K. Tra and Tuan V. Pham, "Human Fall Detection Based On Adaptive Background Mixture Model and HMM," International Conference on Advanced Technologies for Communications, pp. 95 - 100, Oct 2013.

[5] X. Dai, M. Wu, B. Davidson, M. Mahoor and J. Zhang, "Image-based Fall Detection with Human Posture Sequence Modeling," IEEE International Conference on Healthcare Informatics, pp. 376-381, Sep 2013.

http://dx.doi.org/10.1109/ichi.2013.52

[6] J. Willems, G. Debard, B. Bonroy, B. Vanrumste, T. Goedemé, "How to detect human fall in video? An overview", International Conference on Positioning and Context-Awareness, Antwerp Belgium, May 2009.

[7] S. Belongie, J. Malik, and J. Puzicha, "Shape matching and object recognition using shape context," IEEE Transactions on Pattern Analysis and Machine Intelligence, pp. 509-522, 2002. http://dx.doi.org/10.1109/34.993558

[8] R.C. Gonzalez, R.E. Woods, and S.L. Eddins, "Digital Image Processing Using MATLAB", Pearson Education, Inc. and Dorling Kindersley Publishing Inc., ISBN: 81-7758-898-2, 2004.

[9] C. Rougier, J. Meunier, A. St-Arnaud and J. Rousseau, "Robust Video Surveillance for Fall detection Based on Human Shape Deformation," IEEE Trans. on Circuits and Systems for Video Technology, vol. 21, pp. 611-622, 2002. http://dx.doi.org/10.1109/TCSVT.2011.2129370

[10] H.W. Kuhn, "The Hungarian method for the assignment problem," Naval Research Logistic Quarterly, pp. 2:83-97, 1955. 